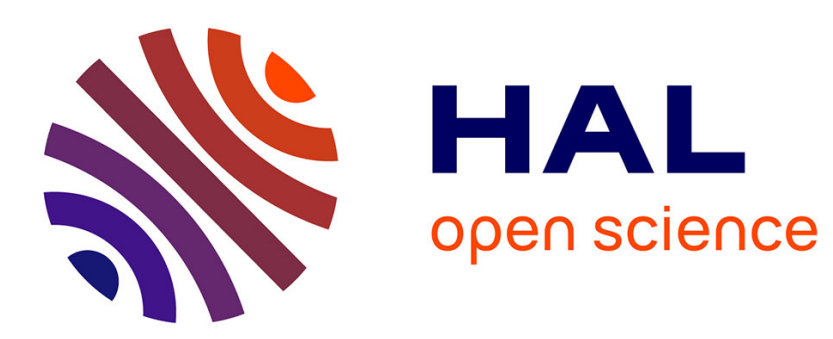

\title{
Le rituel de Khalo Bhut, l'esprit noir : ou comment créer une présence pour mieux la replacer à distance
}

\author{
Grégoire Schlemmer
}

\section{To cite this version:}

Grégoire Schlemmer. Le rituel de Khalo Bhut, l'esprit noir: ou comment créer une présence pour mieux la replacer à distance. Autels et aires sacrificielles: modes de mise en présence et formes d'approche des instances. , Brepols, pp.97-110, 2010. hal-01292251

\section{HAL Id: hal-01292251 \\ https://hal.science/hal-01292251}

Submitted on 28 Mar 2016

HAL is a multi-disciplinary open access archive for the deposit and dissemination of scientific research documents, whether they are published or not. The documents may come from teaching and research institutions in France or abroad, or from public or private research centers.
L'archive ouverte pluridisciplinaire HAL, est destinée au dépôt et à la diffusion de documents scientifiques de niveau recherche, publiés ou non, émanant des établissements d'enseignement et de recherche français ou étrangers, des laboratoires publics ou privés. 
- Bibliothèque de l'Ecole des Hautes Etudes

Sciences Religieuse $\square \mathrm{N}^{\circ} 138$

\section{ARCHITECTURER L'INVISIBLE}

\section{Autels, ligatures, écritures}

Sous la direction de

Michel Cartry

Jean-Lous Durant

Renée Koch Piettre

BREPOLS: Turnout (Belgique) 2010 


\title{
LE RITUEL DE KHĀLO BHŪT, L'ESPRIT NOIR
}

\author{
ou comment créer une présence pour mieux la replacer à distance*
}

\author{
Grégoire SCHLEMMER \\ Institut de Recherche pour le Développement, Paris
}

Les Kulung sont une population d'environ 10000 individus, répartie en une dizaine de villages situés sur les méplats de la vallée de l'Hongu, qui donne sur le massif de l'Éverest, au Népal oriental. Ils appartiennent à l'ensemble Rai qui, avec les autres populations de langue tibéto-birmane, fait partie des groupes que l'on dit « tribaux », par contraste avec les populations indo-népalaises, ou « gens de caste ». Ancien peuple d'essarteurs et de chasseurs, les Kulung vivent aujourd'hui de la culture en terrasse et d'élevage, dans des villages pluri-claniques. Si l'hindouisme, religion dominante du Népal, a influencé les conceptions et les pratiques religieuses des Kulung, ces derniers ont maintenu une organisation religieuse qui leur est spécifique, le muddum. Ce terme, qui signifie littéralement « généalogie » et « mythe », renvoie aussi à l'ensemble du savoir-faire légué par les ancêtres afin de gérer la relation à la pluralité des forces invisibles - que nous nommerons par convention « esprits »-, via les rites. En effet, les Kulung n'ont pas un ensemble précis de catégories discrètes qui permettraient d'ordonner clairement le monde des esprits : ces derniers sont nombreux et changeants, et ils fusionnent ou se différencient selon les contextes rituels lors desquels ces notions apparaissent. Il est donc vain de vouloir englober tous les esprits kulung en un "panthéon ». Dans cette société, c'est donc avant tout par les nombreux rites (chaque maisonnée en exécute environ une trentaine par an) que les esprits se donnent à penser.

Nous observerons un exemple de la manière dont chez les Kulung se construit la présence des esprits, au cours d'un rituel centré sur un autel de circonstance (ce rituel fut enregistré en 1999 et traduit). Distinguant ce qui, pour les Kulung, relève de la « représentation » et ce qui relève de la «manifestation » des esprits, nous essayerons de montrer que plus les esprits sont dits se manifester, c'est-à-dire se rendre présents, moins les Kulung se les représentent, et réciproquement : la manifestation des esprits se réduit en effet à un contact subi dont surgit l'infortune, laquelle entraîne la réalisa-

\footnotetext{
* Je remercie les organisateurs du séminaire de l'EPHE, «Pratiques des polythéismes »-et tout particulièrement Renée Koch Piettre- ainsi que les différents participants de ce séminaire, pour leurs nombreuses remarques. Je remercie aussi Vanina Bouté et Guillaume Rozemberg pour leurs relectures. Cette réflexion s'inscrit aussi dans le prolongement de mon travail de thèse (« Vues d'esprits. La conception des esprits et ses implications chez les Kulung Rai du Népal », thèse de troisième cycle en ethnologie sous la direction de R. Hamayon, Paris 10) et de mon article : « La maladie, c'est les autres : représentation et gestion rituelle de l'altérité chez les Kulung (Népal) », dans C. Culas et F. RoBinNE (éd.), Interethnic Dynamic in Asia. Ethnonyms, Territories and Rituals through the Interethnic Relationships, Singapour, Institute of Southeast Asian Studies.
} 
tion d'un rite qui, en représentant les esprits, c'est-à-dire en les évoquant de multiples manières, a pour effet de les replacer à distance.

\section{Divination et nomination}

Arrivé sur le seuil de la maison de Jit, un ami que je venais visiter à l'improviste, je découvre une certaine agitation dans la maison. Jit, tenant dans ses bras sa petite fille de neuf mois, malade, est assis à côté d'un devin (mobocha, $\mathrm{K}^{1}$ ) qu'il a fait venir pour essayer d'identifier la cause du mal. Après quelques échanges de paroles, notamment sur la santé de la fillette, le devin s'engage dans un rite divinatoire. Sur le bord d'un plat en métal il dépose une poignée de grains de riz qu'il a préalablement mis en contact avec la fillette malade. Tout en marmonnant, il en prend une pincée, la jette au centre du plat, puis se met à répartir les grains par paires. Il s'agit là d'un procédé divinatoire répandu au Népal : tout en invoquant le nom d'un esprit qui pourrait être responsable du mal, le devin observe si les grains de riz qu'il a jetés forment un nombre pair, signe d'une réponse positive à sa question. Le procédé dure un certain temps temps. Il faut en effet effectuer, pour chaque nom d'esprit proposé, trois jetés de riz, car il est nécessaire que, par trois fois, le résultat soit le même pour confirmer la réponse. À cela s'ajoute la longueur de la liste de noms d'esprits à soumettre à ce questionnement, théoriquement tous ceux que le devin connaît. Au bout d'une demi-heure, le devin s'arrête et annonce à Jit et à sa femme que le mal de leur enfant viendrait de Khālo Bhūt, l'Esprit Noir, et des masān, les esprits errants des ghàt, les lieux de crémation. En associant des noms d'esprits à un mal, le devin pose la première pierre à partir de laquelle les figures des esprits en question vont pouvoir se construire.

À ces noms d'esprits, tout un ensemble de représentations discursives sont associées. On dit de ces esprits, que l'on retrouve un peu partout au Népal, qu'ils errent en état de perpétuel mécontentement, et frappent toute personne qui a le malheur de croiser leurs chemins. Tristes, affamés, solitaires, désocialisés, " dé-localisés », envieux, ils peuvent attaquer les humains à tout moment, et s'emparent alors de leurs « âmes » (cf. infra). Les masān sont plus spécifiquement présentés par les Kulung comme des esprits maléfiques de morts indo-népalais, et tout particulièrement des Kāmi, ces membres de la caste des Forgerons. C'est aussi aux Forgerons qu'est associé l'Esprit Noir. Les Forgerons sont présents jusque dans les coins les plus reculés du Népal (comme dans la partie haute de la vallée Hongu, où ils forment l'unique communauté permanente d'Indo-Népalais) et, pour les Kulung, ils semblent repré-

1. Les termes en langue non française sont en italique; un « $\mathrm{K} »$ précise qu'il s'agit d'un terme kulung. Le rituel étant exécuté en népali, les termes issus de cette langue sont plus nombreux. J'ai donc choisi de laisser les mots de cette langue sans indication, sauf en cas de possible confusion; ils seront alors marqués par un « N ». Précisons que le kulung appartient à la famille des langues tibéto-birmanes, alors que le népali fait partie, comme le français, des langues de la famille indo-européenne. La transcription du népali est celle de R. L. Turner, A Comparative and Etymological Dictionary of the Nepali language, Londres 1931. Il n'existe pas de transcription officielle du kulung, et la mienne est à prendre de manière tout à fait indicative. Les noms de groupes sont donnés ici sous une forme invariante, sauf lorsqu'ils sont traduits par une fonction (les Forgerons) ou lorsqu'ils sont d'usage courant en français (les Brahmanes). 
senter l'idéal-type des étrangers; d'autre part, ils forment une caste de bas statut, et l'association péjorative entre les membres de cette caste et les esprits maléfiques que sont les masān reflète le mépris dont ils sont l'objet, mais peut-être aussi leurs liens aux dangereuses forces telluriques.

\section{Préparation et choix de l'emplacement}

Le rite étant relativement simple à réaliser, Jit décide qu'il sera effectué immédiatement. Il s'en va alors demander à l'un de ses voisins -qui se trouve être un parentd'accepter de jouer les rôles d'ancien (paliu, K) et d'aide rituel (pekuyop, K), tous deux nécessaires à la réalisation de la plupart des rites. Cet accord obtenu, Jit saisit un poulet de sa basse-cour, prend son enfant dans ses bras, tandis que le devin rassemble les quelques affaires dont il aura besoin, et la petite troupe s'apprête à partir en direction d'un ruisseau situé à proximité de leurs maisons. Je demande alors si je peux les suivre; il ne s'agit là que d'une formalité, car un rituel n'a généralement rien de privé et tout le monde peut en observer le déroulement.

Nous marchons quelques minutes, et nous arrêtons en un endroit plat et en friche, juste au bord du petit cours d'eau. Le rituel ne peut en effet être réalisé dans une maison ou dans un champ. En revanche, n'importe quel coin de brousse peut faire l'affaire lorsque le rite concerne ces forces maléfiques qui sont dites rôder perpétuellement dans les sortes de non-lieux que forment les espaces incultes -même si l'on recherche de préférence la proximité d'un ruisseau, de manière à pouvoir évacuer les mauvaises influences. Le lieu est désert, mais on voit derrière les arbres dépasser le toit de quelques maisons. En effet, l'habitat kulung est semi-dispersé, c'est-à-dire que, si les maisons sont généralement assez proches les unes des autres (quelques dizaines de mètres), elles sont néanmoins éparpillées au milieu des champs, et il n'existe pas, à proprement parler, d'espace villageois : pour les Kulung, hors des quatre murs de la maison, on est déjà dans l'espace du dehors. Les protagonistes du rituel débroussaillent un peu l'endroit et s'installent, tandis que le devin se prépare à mettre en place le dispositif qui va permettre d'aménager ce lieu ordinaire en un espace momentané de communication avec des esprits. Le dispositif qui va alors se construire sous nos yeux et que nous allons maintenant détailler sera nommé en népali than, « le lieu », « l'endroit », et sum en kulung ${ }^{2}$; nous traduirons ces deux mots par « autel».

\footnotetext{
2. Ce mot de sum désigne aussi curieusement le lieu où l'on laisse reposer la bière. Le lien entre ces deux acceptions (« autel » et « lieu de fermentation ») se situe peut-être au niveau de l'utilisation de graines (centrales dans les deux cas), mais peut aussi venir de l'alchimie qui s'opère au lieu dit sum : les graines y deviennent bière comme la nourriture déposée dans l'autel devient bienfait. Il faut préciser que les termes de than et de sum ne désignent que les constructions temporaires qui sont réalisées pour des rituels non périodiques. Les autels permanents, toujours marqués par une ou plusieurs pierres (plates ou dressées), sont nommés, en kulung, khom. Ce terme désigne littéralement un emplacement qui est de manière permanente le lieu d'une action répétée ou d'une commémoration (mkhom ou mkhammom s'emploie comme verbe signifiant répéter, commémorer, faire une chose en mémoire d'un précédent). En népali, il est généralement traduit par mandir, «temple », mais un ancien me dit qu'il s'agissait plus modestement de chāpa $(\mathrm{N})$, c'est-à-dire de « signes », de « marques », de « traces ».
} 


\section{La mise en place de l'autel}

Le devin -que j'appellerai désormais officiant, car il n'est pas nécessaire d'être devin pour officier à ce rituel ${ }^{3}$ - ramasse dans les alentours une petite pierre qu'il dresse : ce sera l'Esprit Noir. Il lui fabrique aussi un «fanion » (un petit morceau de tissu attaché à une branche, nommé lingo-dhajo, litt. " mât-drapeau de prière »), ainsi qu'une enclume (une petite pierre carrée), un marteau et une cisaille miniatures, faits de brindilles. Puis l'officiant fabrique une croix en bois autour de laquelle des fils sont enroulés en losange ${ }^{4}$, et qu'il m'annonce être une sorte de piège pour les masān. L'officiant ramasse ensuite un peu de terre argileuse qu'il modèle en forme de cône au bout aplati, formant ainsi une grossière figurine en terre (naksāa). Au sommet du cône, il incruste sept petits boudins de terre qui représentent des flammes : c'est l'Esprit Torche, Ranko bhüt. Cet esprit se repère le plus souvent la nuit, lorsque l'on voit, sur des parois rocheuses inaccessibles à l'homme, de petites lumières scintiller tout en se déplaçant; on dit en effet que l'Esprit Torche porte des flammes sur ses épaules. L'officiant modèle enfin une seconde figurine, plus petite, que l'on dit être l'«assistant» (dhole) de l'Esprit Torche. Si l'Esprit Noir et les masān ont été désignés comme responsables de la maladie de l'enfant, le rituel implique donc la figuration d'autres esprits, et on verra que d'autres d'esprits encore seront mentionnés dans les incantations. En effet, au niveau de l'action rituelle, la plupart de ces esprits liés au dehors semblent former un ensemble indistinct : un rite ayant pour finalité d'écarter l'un d'eux en nommera toujours plusieurs dans le même temps.

Les éléments de l'autel renvoient donc à trois types d'esprits : l'Esprit Noir, l'Esprit Torche et son assistant, et les masān, respectivement associés à une pierre, à des figurines en terre et à une croix de fils et de bois. Simples matières tirées du lieu, les pierres et la terre s'en extraient par la forme et la disposition particulière qui leur sont données (la première est dressée, la seconde est malaxée), pour devenir figures des esprits, les représentant (les figurines en terre sont nommées naksāa, ce qui signifie littéralement « dessin », «schéma ») et les établissant (la pierre est nommée par le terme népali sthāpanā, qui signifie littéralement « fixer », « établir », 《 fonder »). Représentations des esprits, elles n'en sont néanmoins pas des «présentifications » : la pierre n'est pas l'esprit, mais un support potentiel (« c'est comme une piste d'atterrissage », me dit un jour un Kulung), et à la fin du rituel, elle sera abandonnée et perdra son statut de support. Ce décalage entre la représentation et ce qu'elle est supposée représenter est aussi marqué par le fait que, lors de certains rituels (comme ceux de protection du bétail, qui impliquent construction simultanée d'une dizaine

3. Si une consultation divinatoire nécessite que l'on soit devin (selemop K), c'est-à-dire que l'on ait été «élu » en rêve ou à cause d'infortunes par un esprit électeur, de nombreux rituels sacrificiels peuvent être réalisés par n'importe quel « ancien » (paliu $\mathrm{K}$ - terme qui désigne tout homme versé dans la connaissance de la tradition et des rituels). Ancien ou devin, en tant qu'officiant, celui qui réalise le rituel sera nommé mobocha $(\mathrm{K})$, ou dhāmi $(\mathrm{N})$.

4. Ces losanges de fils évoquent ceux, du même type ou plus complexes, nommés mdos ou gnam$k h a$, que l'on retrouve fréquemment dans les rituels tibétains dits « de rachat », et qui, représentant un diagramme de l'univers ou un jardin du paradis, attirent les démons dans leur piège. 
d'autels), il n'y a ni figurine, ni pierre dressée : les fanions, qui opère comme des marqueurs des esprits dédicataires, trônent seuls.

On notera aussi la matérialisation d'attributs des esprits, que ce soit de leur nature physique, comme les flammes de l'Esprit Torche, ou de ce qui est supposé former leur activité - mais qui forme aussi ici un attribut de caste-comme le marteau, l'enclume et la pince (en miniature, comme tout ce qui est représenté); ils viennent spécifier les supports d'esprits mis en place. À ces attributs s'ajoutent des éléments qui, tout en pouvant particulariser les esprits, relèvent des dons qui leur sont faits. Ainsi, sur chacune de ces trois figurines, l'officiant place un peu de poudre de vermillon (sindur), et leur fabrique une «lampe », c'est-à-dire un petit morceau de tissu trempé dans du beurre clarifié (directement posé sur les statuettes d'argile ou coincé dans un petit morceau de bois fiché en terre) qui, allumé, dégagera un doux fumet que l'on dit être apprécié des esprits. Pour les trois figurines, l'officiant dispose une assiette en feuille (dunu), dans lesquelles sera déposée la part des esprits (bhog); il y met d'emblée quelques grains de riz préalablement lavés. L'officiant place enfin deux pierres plates sur lesquelles il brûlera de l'encens, composé de bouts séchés de rhododendron (sunpātibairongpāti) que les hommes ramassent dans les hauteurs.

La phase de mise en place de tous ces éléments, qui prend bien une vingtaine de minutes (presque autant de temps que la récitation des incantations), peut être considérée comme partie intégrante du rituel. Le rituel est ici actions sans paroles -en contraste avec la partie suivante du rituel, qui se construit surtout en paroles, les actions, on le verra, y étant réduites. Ces deux phases du rituel obéissent néanmoins à la même logique orthopraxe ${ }^{5}$ : l'exactitude est requise lors de la préparation de cet agencement d'éléments que l'on peut nommer « autel »; tout doit être conforme à la tradition, sous peine de non effectivité du rituel. Car c'est la mise en place rigoureuse de tous ces éléments qui va créer le dispositif capable de faire apparaître, sur une portion d'espace, un lieu possible de communication et d'échange avec ces «êtres » invisibles.

\footnotetext{
5. Le caractère orthopraxe du rituel explique notamment que la présence d'un tiers, tel l'ethnologue, ne change pas grand chose à la réalisation d'un rituel. Il explique aussi le désintérêt éventuel des participants eux-mêmes : ainsi, lors de ce rituel, l'ancien était venu avec sa radio qu'il écoutait de temps en temps, ou discutait avec Jit, tandis que l'officiant prononçait le discours incantatoire, non sans tendre l'oreille lorsqu'il entendait des éclats de rire - ce qui n'excluait néanmoins pas une certaine concentration de sa part, car l'orthopraxie implique aussi que la moindre erreur de réalisation rende le rituel caduc. De même, les discours incantatoires tirent leur effectivité de leur simple prononciation; ils ne sont pas écoutés (même si le sens général des incantations prononcées était connu des participants) et, d'ailleurs, ils n'ont pas de valeur informative pour les humains. J'étais donc bien le seul qui semblait intéressé par ce qui se passait... De cela, on peut conclure que l'effectivité du rituel passe moins par un investissement émotionnel, que par les représentations et les interactions que le rituel impose, par sa réalisation même, à ceux qui le réalisent. Mais on retiendra aussi que cela n'empêche pas que, pour les Kulung, les actions et paroles rituelles ont bien un sens, même si leur compréhension n'est pas nécessaire à leur efficacité et que ce sens peut ne pas être évident. Ainsi la démarche de l'ethnologue qui consiste à tenter de le découvrir est perçue comme légitime, même si parfois futile.
} 


\section{Les incantations}

Le dispositif de communication mis en place, l'officiant va pouvoir entamer la récitation incantatoire. Ces incantations se font en népali, qui n'est pas la langue parlée par les Kulung, mais la langue de la population dominante du pays. Il est d'ailleurs probable que les discours rituels prononcés en népali aient été empruntés aux populations indo-népalaises de la région; cela expliquerait la richesse du vocabulaire utilisé, alors que les Kulung parlent un népali relativement sommaire. Il nous apparaît que cette logique d'emprunt se fonde sur une conception particulière de la maladie et du domaine des puissances invisibles, qui renvoie la cause des maux du côté de l'extériorité : masān et Esprit Noir semblent personnifier la dangereuse altérité de ces populations indo-népalaises, et des groupes étrangers en général. C'est donc dans leur langue, qui est la langue de l'Autre par excellence (notamment du fait qu'elle sert de langue véhiculaire dans toute la région), que ces esprits doivent être chassés.

Les officiants affirment que ces incantations se transmettent de génération en génération, et sont donc immuables. Si cette assertion relève plus d'une pétition de principe que d'une réalité, j'ai cependant pu observer plusieurs rituels exécutés en népali, dont les récitations incantatoires étaient très similaires, à quelques variations près (quelques formules peuvent varier, être omises ou rajoutées). Il me semble donc qu'il existe un type unique de discours incantatoire pour les rituels réalisés en népali, qui varie plus ou moins, selon les officiants, les rituels et les occurrences.

Ce « texte rituel », mémorisé, est prononcé à haute voix, sans interruption, avec un débit très rapide, et de manière rythmée. C'est ce rythme qui en marque le découpage. Le fait que les paroles sont construites comme formant une seule phrase en rend la traduction malaisée (les verbes sont principalement construits comme des participes présents, et les propositions s'enchaînent les unes aux autres). La récitation est en outre composée de formules ou d'ensembles de formules qui sont répétés en boucle, avec parfois quelques variations de termes, ce qui rend le discours très répétitif. C'est pour ces différentes raisons que je ne reproduis pas, dans la description qui va suivre, le texte rituel dans son entier. Il me faut aussi préciser que la répétition des formules fait que tous les éléments sont mêlés dans le discours, même si certaines formules n'apparaissent qu'une ou deux fois, liées à des étapes précises du rituel (ouverture, sacrifice, etc.) C'est donc par l'analyse, et uniquement pour des raisons de clarté, que l'on peut découper le texte rituel en séquences, comme nous nous proposons de le faire.

\section{Les acteurs du rituel}

L'autel mis en place, l'officiant s'assied en face, sur ses talons, tandis que non loin de lui, s'installent l'ancien, Jit et son enfant; on verra que la présence physique du malade bénéficiaire de l'action rituelle est requise, même si sa participation au rituel est minime. L'officiant commence par brûler de l'encens, en versant des pincées de rhododendron séché sur les pierres plates disposées à cet effet et qui supportent quelques braises, jette quelques grains de riz sur l'autel, puis il entame les incantations. 
Il commence par nommer le moment de la communication avec les esprits en question; ceci se résume d'ordinaire à une formule telle que : « nous concertant, nous avons décidé de te rendre culte en ce jour propice (din-bār) de (telle) saison », mais dans le rite ici décrit, la formule se réduit à un laconique : « aujourd'hui ». Le lieu est lui aussi simplement présenté, puisqu'on se contente de dire : « dans cet autel» (thānma). Aussi sommaire soit-elle, cette spécification d'un lieu et d'un temps isole un segment spatio-temporel particulier au sein duquel une certaine forme de communication pourra s'établir.

Le cadre spatio-temporel du rituel étant ainsi posé, l'officiant précise les raisons qui ont motivé l'exécution du rituel : «Toi [l'esprit -en dépit du fait que le rituel implique plusieurs esprits, le pluriel n'est pas marqué, comme si tous ces esprits du dehors étaient ici regroupés, subsumés, en une même entité], rendant ce petit enfant malade, mettant ce jeune enfant en danger, frappant de ton ombre, prenant au piège de ton ombre, étant là, tu as frappé cet enfant, tu l'as fait s'évanouir, tu lui as donné une convulsion »; il précise aussi les symptômes du mal : « mal au cœur, mal au foie, les bras et les jambes sans mouvements », et rappelle que c'est par la divination que les esprits ont été identifiés comme responsables des maux de l'enfant («toi, rendant ce petit enfant malade, voyant la divination te désignant »).

Les participants sont, comme lors de chaque rituel, nommés par leur dapsning (K), leur nom de clan rituel. L'officiant se nomme lui aussi et précise le lien de parenté (réel ou classificatoire) qui l'unit au malade bénéficiaire du rituel (l'officiant dit, en kulung, « mon filleul » et prononce son nom de clan rituel). Les esprits, que les incantations précisent être venus ensemble, « groupés », sont nommés par un ou plusieurs noms, que l'officiant récite de très nombreuses fois, et auxquels il ajoute tout un ensemble de qualificatifs. Dans ce rite, il est fait mention de l'Esprit Noir, de Murkuta (du népali murkațto, esprit qui est dit porter sa tête sous le bras), de l'Esprit Torche, des sorcières (dan்kin̄̄) et des masān. Les masān sont présentés -toujours dans ces incantations rituelles - comme des entités multiples : il y a le masān noir, le masān blanc, ceux du monde (jagat, prithvī), du feu, des précipices (bhir), de la terre ferme (jal-thal). Les sorcières, elles aussi nombreuses, sont présentées comme sept sœurs : celle de l'eau, celle des rivières, celles des castes des Forgerons, des Couturiers, des Brahmanes, des Ksatrya et celle des Tamang ${ }^{6}$. Il est aussi question de « roi des esprits » (karbir), mais il semble qu'il s'agisse ici plus d'un qualificatif que d'un esprit particulier. Ces esprits, présentés comme des êtres « nés d'eux-mêmes au début des temps » (aonlatma utpanna baeko), sont dits « errer dans le monde », « jouer », « traverser » et « se baigner », dans l'eau, les fontaines, les rivières, les lieux de crémation ( $g h \bar{a} t)$, les croisées de chemins, le ciel et la terre. Tous ces qualificatifs, qui œuvrent

\footnotetext{
6. Brahmane et Ksatrya sont des hautes castes. Avec les basses castes des Forgerons et des Couturiers (Damāi $)$, ils forment les principales castes indo-népalaises de la région. Les Tamang sont une population bouddhiste de langue tibéto-birmane de la région. On notera que, peut-être par oubli, cette liste ne comprend pas l'ensemble des populations de la région : il manque ici les Sherpa, une population bouddhiste importante dans la région. Mais le fait qu'aucun groupe rai (Khaling, Thulung, Nachering, etc.) ne soit cité m'apparaît dû au fait que, plus proche des Kulung, ils ne font pas partie de ces dangereux Autres d'où proviennent les sorcières.
} 
comme autant de déclinaisons de l'identité de ces esprits, évoquent le lien qui unit ces derniers à une extériorité, celle du monde sauvage et des autres groupes humains. Ces spécificateurs insistent aussi sur la mobilité de ces esprits (à travers la multiplication des lieux nommés, ainsi que la notion de mouvement induit dans les verbes « errer », « traverser », etc.) On notera enfin la récurrence des espaces interstitiels (qu'ils soient lieux de communication, comme la croisée des chemins, ou frontière, comme les rivières et les précipices).

\section{Description de l'autel, énumération des offrandes et annonce des demandes}

L'espace et le lieu étant déterminés et les protagonistes humains et non-humains étant déclinés, vient le tour des éléments qui forment l'autel. Aux interlocuteurs invisibles, toutes les choses offertes et toutes les actions exécutées doivent être nommées, ce qui fait de cet acte de nomination un élément essentiel des offrandes. Les biens préparés et offerts aux esprits sont désignés : «dressant ta "carte", faisant ton arc et tes flèches, les sept graines, le bétel-noix d'arec, le poivre et les clous de girofle, le vermillon et le khôl, faisant des beignets aux graines de sésame, ta pince, ton marteau, ton enclume, préparant ton drapeau et ton mât, ta mesure de riz cru, ton bélier sacrificiel (? mera) étant posé, toi, préparant un poulet à la crête à neuf dents, à la crête et aux pattes pures, une poule d'or, de l'encens de rhododendron du mont Kailas ». Si les incantations semblent comme reproduire, sous forme de mots, les objets et actes du rituel, la correspondance n'est néanmoins pas complète. Ainsi, dans la liste des offrandes et des autres éléments formant le dispositif rituel, certains sont absents de l'autel : bétel, noix d'arec, poivre, clous de girofle, khôl, etc. Certains éléments peuvent aussi faire l'objet d'une substitution (lors d'un rituel, tel esprit demande une poule noire mais le commanditaire du rituel n'en a que des rouges; il prend une poule rouge et la présentera comme noire à l'esprit), ou être plutôt enjolivés (le poulet, qui était, en l'occurrence, quelque peu déplumé, est vanté pour sa beauté). Le discours ne se contente donc pas de convertir en mots les éléments présents, il vient aussi comme les compléter, les enrichir et les améliorer et, ce faisant, contribue à fonder l'espace rituel.

Cette présentation des offrandes est suivie des requêtes exigées en contrepartie. L'officiant demande protection (raksāa, pichā), « faveurs » et «bienfaits » (bar). Ce terme de bar est important, et l'on entend souvent dire que « faire un rituel, c'est donner bhog contre bar », c'est-à-dire des offrandes contre des bienfaits. La principale demande est plus précisément le retour des âmes et de la santé de l'enfant : l'officiant demande aux esprits auxquels s'adresse le rituel de rendre les âmes qu'ils auraient cachées dans les falaises, les forêts, les rivières, les lieux de crémation, et les croisements des sept chemins. Que, étant « ouverts », les esprits rejettent, de leur main droite et de leur main gauche, les âmes (désignées tout au long du rituel par différents termes népali : hañsa, sir-sāto, sāto-āyu) qu'ils ont volées. L'officiant demande alors aux esprits « de les jeter dans l'autel avec vitalité en faisant la lumière dessus et après les avoir lavées dans l'eau des sept océans », mais aussi qu'ils les ramènent dotées de vitalité $(\bar{a} y u)$, qu'ils les rendent « grandes comme les falaises et brillantes comme des lampes ». Enfin, il demande à ces esprits d'apporter et de donner la vie $(\bar{a} y u)$, la force 
vitale (sai K), la force de volonté (șaikkalpa), et de rendre les âmes grandes comme des falaises et brillantes comme des lampes. La récurrence des termes qui évoquent le mouvement semble vouloir présager le retour, avec les âmes, d'une vitalité, qui contraste avec l'état de prostration du malade et avec sa passivité convenue lors du rituel.

\section{La séquence sacrificielle}

Les offrandes ayant été présentées et les contreparties formulées, il faut maintenant effectuer la transaction : c'est la séquence sacrificielle, que l'on peut décliner en deux temps : le transfert des maux vers la victime sacrificielle et sa consécration, puis sa mise à mort et l'offrande de sang.

Parlant à nouveau du poulet - que l'officiant a pris dans ses mains au moment où il l'a nommé dans l'énumération des offrandes-l'officiant « dédie » (repcham K) l'animal offert. Pour ce faire, il applique sur l'échine du volatile des grains de riz prélevés dans chacune des trois petites assiettes disposées pour les esprits, puis y verse de la bière. Ces grains, la bière, et parfois l'animal lui-même, sont préalablement mis en contact avec le corps du bénéficiaire du rituel -ici la fillette. Ces actes, réalisés lors de chaque rituel, ont une vertu quasi-médicinale : ils sont présentés par les officiants comme ayant pour finalité de « sortir » (galm K, choțaunu $\mathrm{N})$ les maladies du corps du malade, mais aussi, peut-être, de marquer l'identité du bénéficiaire du rite auprès de l'esprit à qui l'animal est offert. Lorsque l'on verse la bière sur l'échine du poulet, l'officiant attend que l'animal s'ébroue, signe -dit-on-que le sacrifice est accepté par l'esprit auquel il est adressé. Cette action est très importante et un poulet qui ne se secoue pas est source d'anxiété pour les participants. Mais l'animal finit toujours par le faire, car, s'il le faut, l'officiant l'aspergera de bière jusqu'à l'obtention du résultat escompté. La « dédicace » a donc pour finalité d'opérer un lien entre le commanditaire du rituel et l'animal, d'extraire les maladies du corps du malade, mais aussi de s'assurer que le sacrifice est accepté.

Après la « dédicace », l'officiant enflamme les bouts de tissu qui font office de lampes, et met à mort l'animal. Comme lors de tous les rituels réalisés en népali, les animaux ont le cou tranché : la machette est coincée, tranchant vers le haut, entre les jambes de l'officiant, et le cou du volatile est pressé sur la lame. Cette mise à mort libère le souffle et fait couler abondamment le sang (il s'agit, littéralement, d'une « offrande de sang », rakta dān). L'officiant presse entre ses doigts le cou du volatile pour retenir le sang, l'approche des pierre et figurines d'esprits et de l'autel, puis relâche sa pression, faisant ainsi couler abondamment le sang et réjouissant de la sorte les esprits qui sont dits en être avides. Surtout, les anciens disent que les « âmes » reviennent via le sang de l'animal sacrifié. Il faut au moins une goutte sur chaque support d'esprit, mais aussi sur le marteau et l'enclume, comme si elles avaient le même statut que les figurines. Le corps sanguinolent du volatile est alors éloigné (afin de ne pas éclabousser), l'officiant prend sa tête et la dépose dans l'assiette en feuille de l'Esprit Noir. Il dispose aussi une plume à côté de chaque figurine d'esprit ainsi que sur l'enclume. 
L'animal décapité et le sang offert, l'officiant mentionne à nouveau la finalité de l'échange : spécifiant que l'animal a bien été offert, il rappelle toutes les autres offrandes présentes et répète le but recherché : la guérison de l'enfant. Le sacrifice est clairement conçu comme une substitution. Si ce ne fut pas le cas lors de ce rituel, une fois un sacrifice effectué, l'officiant prononce généralement ces paroles : « aujourd'hui, nous, humains (manuwā : les humains, du point de vue des esprits), en échange du corps, un corps a été donné, en échange du souffle, un souffle a été donné » (jiuka badali, jiu dieko cha, sāska botli, sās dieko cha).

\section{Le rappel des âmes}

Le sacrifice réalisé, il est alors possible d'« appeler les âmes » (law kachom K). La notion de law, que je traduis par commodité par « âme », n'a rien à voir avec la conscience, ni même avec la pensée ou les émotions. Les Kulung conçoivent les âmes (car les humains en possèdent plusieurs) comme des sortes de principes actifs qui animent le corps, permettant le mouvement et l'action. Les âmes forment une réalité assez obscure et abstraite à laquelle ils ne se réfèrent généralement qu'en cas de problème, c'est-à-dire quand elles sont supposées être absentes. Car les âmes sont plus ou moins indépendantes des corps qu'elles habitent et qu'elles peuvent quitter, à la suite d'un effroi, d'une chute, ou lorsque, ayant quitté le corps pour se promener et jouer, elles se sont fait capturer par un des esprits errants. Les âmes semblent donc conçues avant tout comme une énergie humaine qui sert de pâture aux esprits. Notons enfin qu'aucune connotation de faute n'est liée à la perte des âmes : celles-ci sont capturées, telles des proies, par les esprits errants qui cherchent à se nourrir. Lorsque des âmes ont été volées, le rappel des âmes constitue, avec l'éloignement des infortunes hors du corps du malade, l'objectif principal du rituel.

Tout en tapant sur un bol métallique qui fait office de cymbale ${ }^{7}$ et en aspergeant le patient de bière, l'officiant et l'ancien qui est venu aider à l'exécution du rituel demandent alors aux âmes de revenir. Composantes de la personne, c'est toujours en kulung qu'il faut s'adresser à elles, même s'il s'agit d'un rituel en népali. Les deux hommes énumèrent une liste plus ou moins standardisée de noms d'âmes qui auraient été cachées dans la forêt et que l'on « arrache » (horsum, K) aux mauvais esprits. Ainsi appelées, les âmes sont alors dites venir d'elles-mêmes vers l'autel ${ }^{8}$.

Les âmes étant censées tournoyer autour de l'autel, l'officiant scrute l'invisible et, d'un geste sec, attrape du poing ce qu'il dit être une âme (law lapam K). Il reni-

7. Le bruit métallique (khapci samlo samlomoto $\mathrm{K}$ ), dont le terme kulung évoque aussi le son de la richesse (khapci étant un terme utilisé comme synecdoque pour désigner la richesse en général) attire les âmes et leur plaît, tandis qu'il est dit faire fuir les mauvais esprits (kuyama-mayama K) qui peuvent être présents lors de la venue des âmes.

8. Cette fonction de l'autel comme « capteur d'âmes » est particulièrement probante lorsqu'il s'agit d'autels faits d'aspersoirs, c'est-à-dire de bambous effilochés (soit aux deux extrémités, soit à l'une) qui servent au devin à laver les corps et les âmes, mais aussi à les protéger contre les attaques magiques. Ces autels en aspersoirs sont généralement construits pour les rituels aux esprits forestiers, eux-aussi conçus comme de grands dévoreurs d'âmes. 
fle alors son poing serré, laisse un bref instant l'âme s'échapper, puis la rattrape prestement et la renifle encore; il s'agit d'un examen de l'âme (law cetam K), qui a notamment pour but d'identifier à qui elle appartient (l'officiant dit « l'examinant par l'odeur, cela est-il bien l'âme de cette fille cadette du clan X? ») Ces opérations sont effectuées grâce à son pouvoir ancestral (yum K), dont les Kulung disent souvent qu'il lui donne le flair d'un chien de chasse, soulignant ainsi l'aspect cynégétique du rituel. De son autre main, il verse de l'eau sur son poing fermé; il s'agit de nettoyer l'âme. Puis il lui fait réintégrer le corps de son propriétaire en la jetant dans sa bouche (on la lui « fait manger », law poyem K), et en faisant boire de la bière au malade. Enfin, il la fixe en appuyant son pouce ou des grains de riz pris dans l'autel -et qui doivent être ensanglantés par le sang du sacrifice- entre les deux yeux de la personne. Il demande enfin à cette âme d'être forte et de ne plus quitter dorénavant le corps de son propriétaire. On notera un certain parallélisme entre le fait d'appliquer des grains de riz et de verser de la bière sur l'animal sacrifié, et le fait de faire boire de la bière au patient et d'appliquer entre ses yeux des grains de riz au moment de lui faire ingurgiter ses âmes, ce qui tend à renforcer l'association entre le sacrifiant et la victime sacrificielle.

La première moitié du rituel est alors terminée. La transition avec la seconde partie du rituel est marquée par une pause, que l'on consacre à la préparation de la viande. Le père de la malade et l'ancien allument un petit feu, au-dessus duquel ils passent le volatile, puis ils le nettoient de ses restes de plumes calcinées. Les trois hommes profitent aussi de cette pause pour boire de la bière, et discuter de choses diverses.

\section{La clôture du rituel et le traitement des restes}

La cuisson terminée, la deuxième partie du rituel peut commencer. De l'encens est remis à brûler et les invocations sont répétées : l'officiant reformule les raisons d'être du rituel, l'ensemble des offrandes effectuées, et les demandes. Cette séquence, qui ne fait pas intervenir de nouvelles actions rituelles -excepté le fait que, lorsqu'il parle de l'enclume, l'officiant prend une braise avec la pince, la pose sur l'enclume, et frappe dessus avec le marteau - n'en est pas moins presque aussi longue que la première. La tête du poulet qui était déposée dans l'autel est alors reprise; l'officiant se saisit également du corps cuit du poulet et prélève sur différentes parties (pattes, ailes, croupion, etc.) de minuscules bouts qu'il dépose dans les trois assiettes destinées aux esprits. Il met ensuite fin à ses incantations, sans que gestes ou mots évoquent une quelconque idée de clôture. Puis il se lève, et le rituel est fini; tous s'apprêtent alors à partir. L'autel est donc abandonné tel quel après que les offrandes -hormis celles qui sont déposées dans les trois assiettes- ont été récupérées. Tant que l'autel restera en place, tant que subsisteront des traces de la transaction qui s'est effectuée, on l'évitera, comme si le dispositif mis en place pour la réalisation du rituel portait des traces de la communication dangereuse qui s'y était opérée.

Vient ensuite la consommation, entre les participants du rituel, des aliments qui ont été offerts aux esprits et dont seules d'infimes parties (proportionnelles à la matérialité des esprits?) leur ont été laissées. Lors des rites comme celui-ci, adressés à 
des esprits errants du dehors, ce repas doit normalement se tenir sur place; on dit que l'on ne doit pas ramener ce type de nourriture chez soi. Pourtant la chose se pratique couramment, et sans même se concerter, les trois hommes décidèrent de partir consommer ce poulet dans la maison de Jit. On notera qu'il reste par contre impératif que la viande soit au moins cuite sur place.

Cette nourriture - principalement composée de la viande du sacrifice- est nommée par le terme népali de prasād, c'est-à-dire des offrandes chargées de bienfaits, et la consommation des aliments qui formeront le «festin rituel » est conçue comme une appropriation immédiate des bienfaits du rituel (même si leurs effets sont généralement différés). Ces aliments sont en effet comme chargés de la puissance des esprits pour avoir été en contact avec eux, et l'on dit à leur sujet qu'ils forment le jhutō de ces esprits - jhutō pouvant se traduire par « restes », « déchets », « souillures ». Dans le système des castes, structuré par une hiérarchie des groupes humains en fonction de leur degré de pureté, l'échange des substances corporelles est le principal vecteur de pollution d'un individu à un autre. Un aliment qui entrerait en contact avec le corps d'un individu (on mange avec les mains) devient impur pour les autres; on le nommera alors juthō. C'est donc une sorte de commensalité entre hommes et esprits qui marque la clôture du rituel. C'est la raison pour laquelle les Kulung disent souvent que manger ou boire de la bière d'un rituel, c'est en quelque sorte participer au rituel. Ce festin rituel contraste avec les pratiques alimentaires quotidiennes par son degré de convivialité, et par la vitalité censée être retrouvée grâce à la consommation de la viande.

\section{Conclusion}

Pour conclure cette présentation, reprenons de manière synthétique les différents éléments qui ont à voir avec le problème qui nous préoccupe ici, à savoir la mise en présence des invisibles au travers de ce rituel, centré autour d'un autel construit pour l'occasion. Toute l'opération débute par la venue d'un mal, d'une infortune. C'est via le processus divinatoire -dont le questionnement est toujours du type : « est-ce tel esprit ou celui-là? »- qu'est préalablement activée la notion d'esprit, qui renvoie ici à la cause d'un mal. Si donc la divination est présentée par les Kulung comme ayant pour finalité de dévoiler une ou plusieurs forces invisibles, elle est pour nous, bien au contraire, l'élément qui les instaure; elle les crée en les nommant. Mais les esprits n'apparaissent alors que comme source de maux; leur manifestation se réduit à un contact subi duquel surgit l'infortune.

C'est le rituel, réalisé en accord avec le résultat de la divination, qui vient spécifier les esprits. Certains éléments de l'autel forment les attributs de ces esprits (tels l'enclume et les outils). Le temps du rite, ces esprits errants reçoivent un support matériel qui vient les fixer momentanément. L'autel œuvre comme une sorte de porte qui donne accès à ces êtres invisibles; c'est le lieu de la communication, le seul lieu autorisé de l'échange. C'est à travers lui que vont s'effectuer les offrandes, et l'autel est avant tout conçu comme une table à manger. L'aspect alimentaire du rituel est ici central. Il s'agit de récupérer les âmes, cet aspect énergétique de l'homme que des esprits ont pu voler pour se nourrir ou pour s'en faire un compagnon de jeu, contre 
des paroles, le souffle et le sang de l'animal sacrifié, qui viennent combler cette insatisfaction alimentaire et affective chronique, caractéristique des esprits.

Lors des rituels, donc, tout évoque les esprits, tout les définit : le moment de la performance, les circonstances qui la motivent, les lieux où ils se déroulent, leurs commanditaires, les officiants qui les exécutent, les éléments nécessaires à leur réalisation, les langues parlées, les mots prononcés et les gestes effectués, la nourriture offerte, ce qui est demandé, etc. L'analyse comparative et détaillée de chacun de ces aspects dans l'ensemble des rites effectués par les Kulung montre comment le déroulement des rituels construit progressivement, en creux, un portrait des esprits, et de ceux qui leur rendent un culte.

Pour autant, les esprits en question sont dits ne quasiment pas se manifester lors des rituels. Ils sont certes supposés être impliqués, et ils sont à l'origine de l'alchimie qui opérera, notamment via le festin rituel. Mais leur manifestation est minime (l'ébrouement de l'animal), souvent indirecte (le retour des âmes), et leur action différée (les effets du rite ne seront connus qu'ultérieurement). C'est que le rituel semble avoir pour finalité de faire «sortir » la puissance qui, d'un mal logé dans le corps du malade, irait comme investir un support extérieur : l'autel et ses figurines, via l'animal sacrificiel. Le lieu où la communication s'est établie reste alors comme « imprégné » de la présence des esprits, ce pourquoi on évite sa fréquentation jusqu'à la désagrégation de l'autel. Il s'agirait donc moins de faire venir les esprits que de les faire partir, et les seuls commentaires du rituel qui évoquent une «présence » désignent celle des âmes, que l'on cherche, elles, à faire revenir. Il s'agit bien de donner une forme à l'invisible pour en réduire la présence envahissante (selon une interprétation proposée dans le présent volume par Danouta Liberski), ce qui contraste avec certaines configurations du rite grec, où le rituel se présente comme une manœuvre de captation, et où les instances peuvent être opératrices du rite, y agir.

Partant d'une simple équation « infortune = esprit(s) », la divination rend manifestes les esprits supposés être à l'origine du mal, et le rituel établit ensuite une relation avec eux. Paradoxalement, ce double acte de détermination (via la divination) et de communication (via le rituel) recréera la nécessaire distance entre les hommes et les esprits. Lors du rituel, les esprits n'existent plus qu'en creux; ils ne sont plus, en quelque sorte, que les destinataires idéaux de la communication rituelle. La relation doit donc exister, mais être limitée, car si elle est nécessaire, elle est aussi dangereuse.

\section{Résumé}

À travers la description minutieuse d'un petit rituel réalisé par les Kulung Rai de l'Himalaya népalais, l'auteur tente de saisir une des modalités rituelles de la mise en présence des invisibles (ou « esprits») autour d'un autel de circonstance. En plaidant pour la nécessité de distinguer ce qui, pour les Kulung, relève de la «représentation» de ce qui relève de la "manifestation» des esprits, l'auteur tente de montrer que, dans ce rituel, représenter les esprits n'a pas pour finalité de les rendre présents, mais au contraire de replacer à distance ces présences envahissantes. 


\section{Abstract}

Through the meticulous description of a ritual performed by Kulung Rai (Nepal, Himalayas) on a temporary altar, the author tries to understand one of the ritual modality of mise en présence of invisibles (or "spirits»). Pleading for the necessity to distinguish what, for the Kulung, is a "representation " from what is a "manifestation » of spirits, the author tries to show that this ritual does not try to make spirits present, but to put these intrusive presences away.

Schéma de l'autel (thān) du rituel de Khālo Bhūt

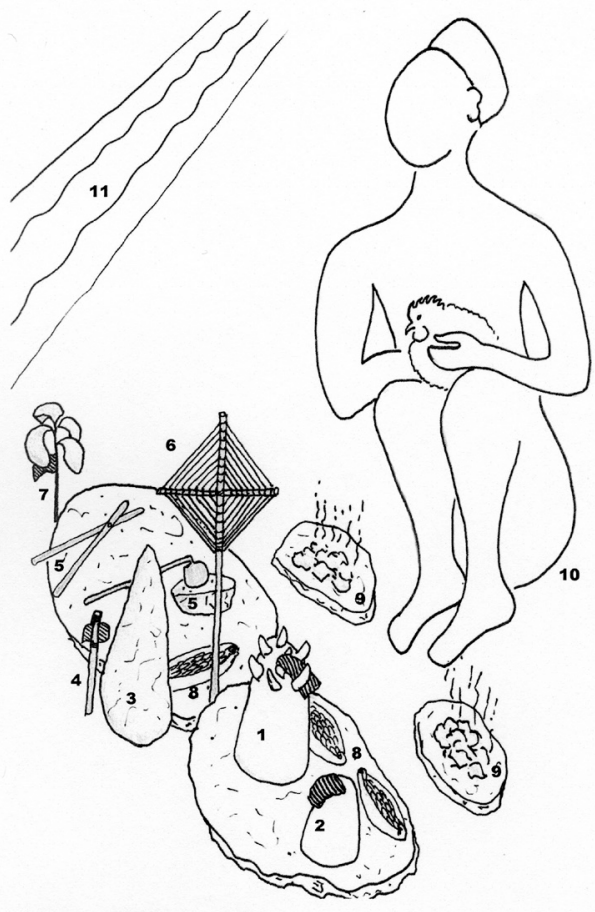

Le schéma n'est pas à l'échelle

1 Figure en terre (naksā) représentant l'Esprit torche (Ranko bhüt) et sa lampe

Figure en terre (naksā) représentant l'Assistant (Dhole) et sa lampe

Pierre (sthāpanā) représentant l'Esprit Noir (Khālo Bhüt)

Lampe (tissu imbibé de beurre)

Marteau, enclume et pince miniature

Croix servant de pièges aux masān

Fanion ou "mât-drapeau de prière" (lingo-dhajo)

Assiette en feuille pour offrande (dunu)

Pierres encensoirs

L'officiant et le poulet sacrificiel

11 Ruisseau 